\title{
Artificial intelligence to classify ear disease from otoscopy: A systematic review and meta-analysis
}

\author{
Al-Rahim Habib ${ }^{1}$, Majid Kajbafzadeh ${ }^{1}$, Zubair Hasan ${ }^{2}$, Eugene Wong², Hasantha \\ Gunasekera $^{1}$, Christopher Perry ${ }^{3}$, Raymond Sacks ${ }^{4}$, Ashnil Kumar ${ }^{1}$, and Narinder Singh ${ }^{5}$ \\ ${ }^{1}$ The University of Sydney \\ ${ }^{2}$ Westmead Hospital \\ ${ }^{3}$ Brisbane ENT \\ ${ }^{4}$ Sydney Medical School \\ ${ }^{5}$ University of Sydney/ Westmead Hospital
}

November 24, 2021

\begin{abstract}
Objective: To summarize the accuracy of artificial intelligence (AI) computer vision algorithms to classify ear disease from otoscopy. Methods: Using the PRISMA guidelines, nine online databases were searched for articles that used AI methods (convolutional neural networks, artificial neural networks, support vector machines, decision trees, k-nearest neighbors) to classify otoscopic images. Diagnostic classes of interest: normal tympanic membrane, acute otitis media (AOM), otitis media with effusion (OME), chronic otitis media (COM) with or without perforation, cholesteatoma, and canal obstruction. Main Outcome Measures: Accuracy to correctly classify otoscopic images compared to otolaryngologists (ground-truth). The Quality Assessment of Diagnostic Accuracy Studies Version 2 tool was used to assess the quality of methodology and risk of bias. Results: Thirty-nine articles were included. Algorithms achieved 90.7\% (95\%CI: 90.1 - 91.3\%) accuracy to difference between normal or abnormal otoscopy images in 14 studies. The most common multi-classification algorithm (3 or more diagnostic classes) achieved 97.6\% (95\% CI: 97.3.- 97.9\%) accuracy to differentiate between normal, AOM and OME in 3 studies. Compared to manual classification, AI algorithms outperformed human assessors to classify otoscopy images achieving $93.4 \%$ (95\% CI: $90.5-$ $96.4 \%$ ) versus $73.2 \%$ (95\%CI: $67.9-78.5 \%$ ) accuracy in 3 studies. Convolutional neural networks achieved the highest accuracy compared to other classification methods. Conclusion: AI can classify ear disease from otoscopy. A concerted effort is required to establish a comprehensive and reliable otoscopy database for algorithm training. An AI-supported otoscopy system may assist health care workers, trainees, and primary care practitioners with less otology experience identify ear disease.
\end{abstract}

\section{Hosted file}

Artificial intelligence for otoscopy_Systematic Review and Meta Analysis_17.11.21.docx available at https://authorea.com/users/447768/articles/546714-artificial-intelligence-toclassify-ear-disease-from-otoscopy-a-systematic-review-and-meta-analysis 

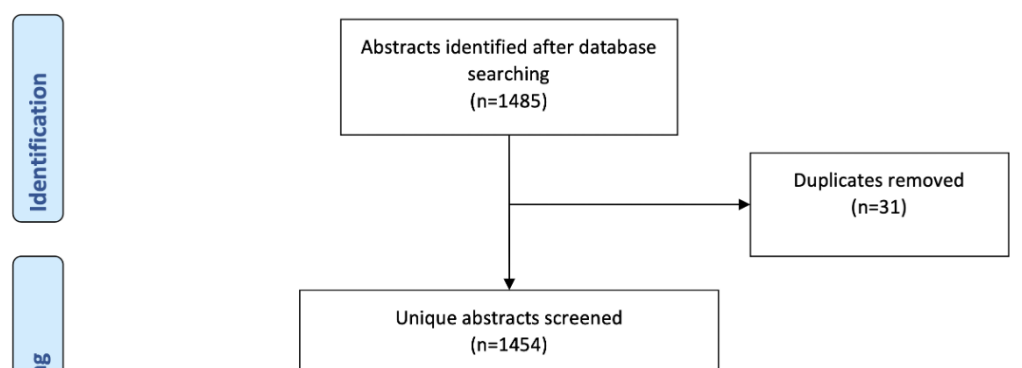

竞

$(n=1454)$
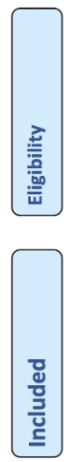

Articles eligible for data extraction

류ํ

Studies eligible for full-text

review

$(n=54)$

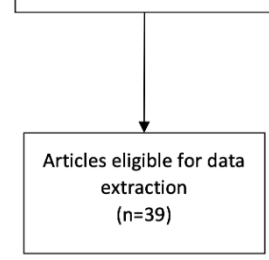

Patient Selection

Index Test

Reference Standard

Flow and Timing 202

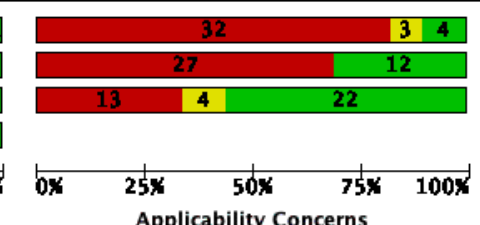

0x 25x

Risk of Bias

Applicability Concerns

Hkgh

$\square$ Unclear

$\square$ Low 


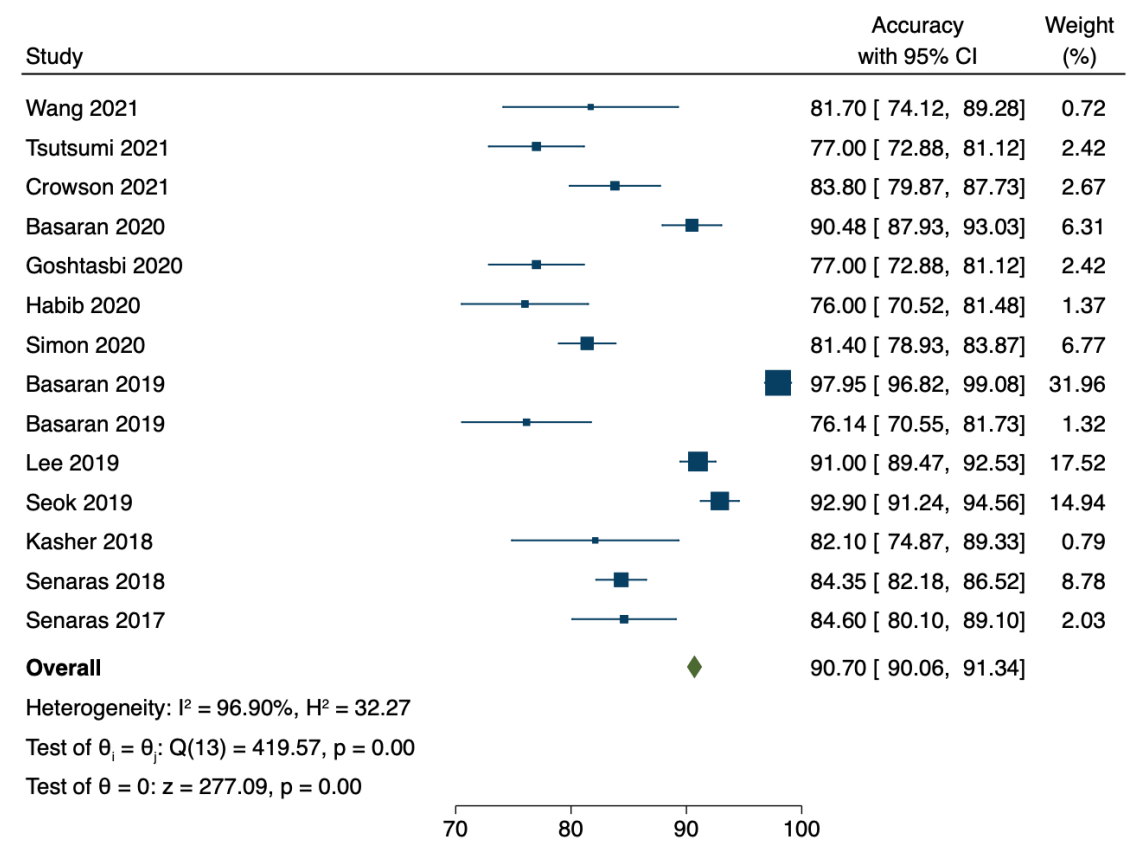




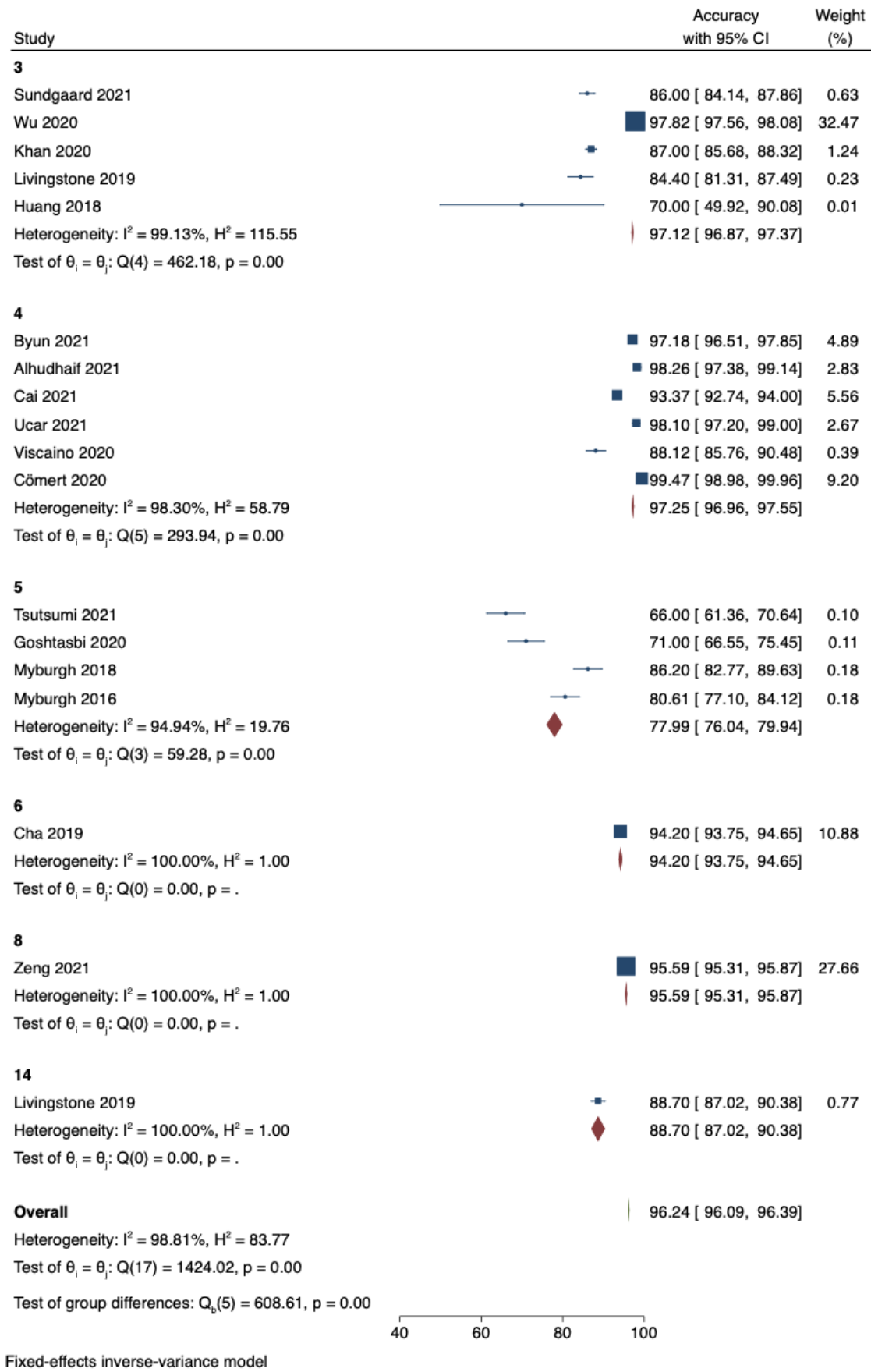




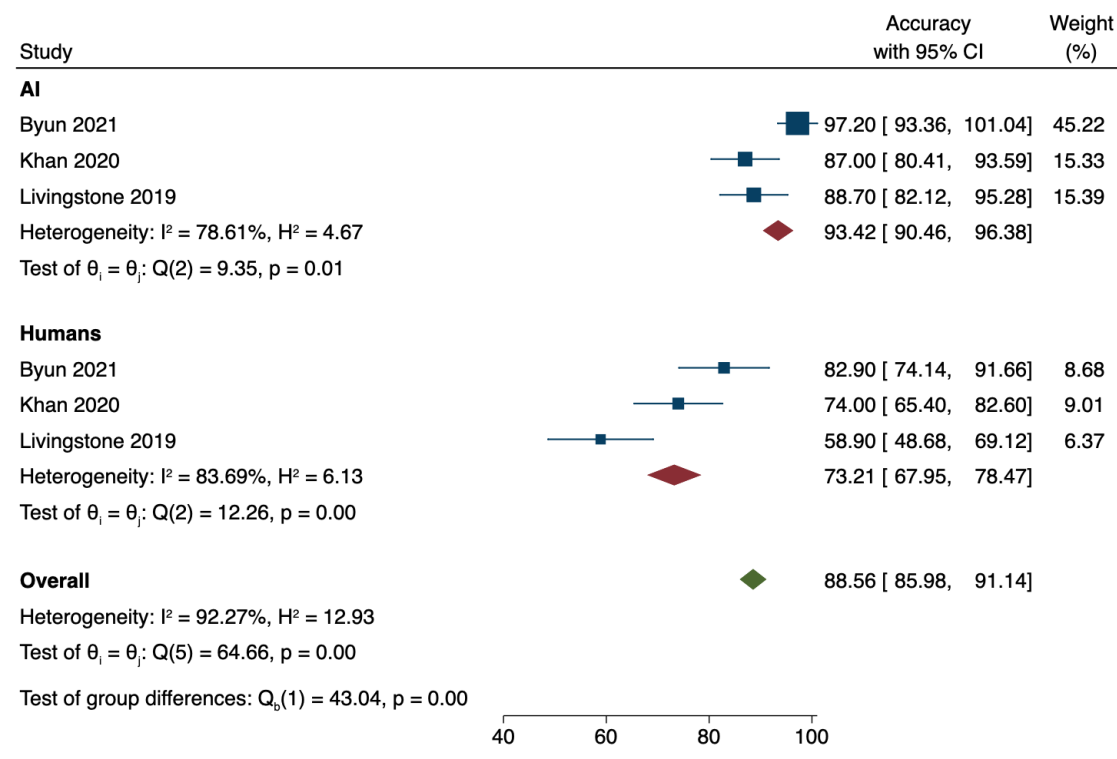

\section{Hosted file}

Table 1.docx available at https://authorea.com/users/447768/articles/546714-artificialintelligence-to-classify-ear-disease-from-otoscopy-a-systematic-review-and-meta-analysis 